\title{
Targeting polyelectrolyte networks in purulent body fluids to modulate bactericidal properties of some antibiotics
}

This article was published in the following Dove Press journal: Infection and Drug Resistance

\author{
Robert Bucki ${ }^{1, *}$ \\ Bonita Durnaś2,* \\ Marzena Wątek ${ }^{2,3}$ \\ Ewelina Piktel' \\ Katrina $\mathrm{Cruz}^{4}$ \\ Przemysław Wolak ${ }^{2}$ \\ Paul B Savage ${ }^{5}$ \\ Paul A Janmey ${ }^{4}$
}

'Department of Microbiological and Nanobiomedical Engineering, Medical University of Białystok, Białystok, ${ }^{2}$ Department of Microbiology and Immunology, The Faculty of Health Sciences of the Jan Kochanowski University in Kielce, ${ }^{3} \mathrm{Holy}$ Cross Oncology Center of Kielce, Kielce, Kielce, Poland; ${ }^{4}$ Department of Physiology, Institute for Medicine and Engineering, University of Pennsylvania, Philadelphia, PA, ${ }^{5}$ Department of Chemistry and Biochemistry, Brigham Young University, Provo, UT, USA

*These authors contributed equally to this work

Correspondence: Robert Bucki Department of Microbiological and Nanobiomedical Engineering, Medical University of Białystok, Mickiewicza 2c, Białystok 15-222, Poland

Tel +48 857485493

Fax +48 857485416

Email buckirobert@gmail.com

\begin{abstract}
The response of the human immune system to most bacterial infections results in accumulation of neutrophils at infection sites that release a significant quantity of DNA and F-actin. Both are negatively charged polyelectrolytes that can interact with positively charged host defense molecules such as cathelicidin-delivered LL-37 peptide or other cationic antibiotic agents. Evaluation of the ability of bacterial outgrowth (using luminescence measurements or counting colony-forming units) to form a biofilm (quantified by crystal violet staining) and analysis of the structure of DNA/F-actin network by optical microscopy in human pus samples treated with different antibiotics in combination with plasma gelsolin, DNAse 1, and/or polyaspartic acid revealed that bactericidal activity of most tested antibacterial agents increases in the presence of DNA/F-actin depolymerizing factors.
\end{abstract}

Keywords: antibiotic activity, polyelectrolyte network, depolymerizing factors, cathelicidin, ceragenins, DNase 1, cystic fibrosis

\section{Introduction}

Treatment of chronic infections associated with the production of purulent secretions is often challenging. An example of such a situation is pulmonary exacerbation in cystic fibrosis $(\mathrm{CF})$ patients where strong, neutrophilic endobronchial inflammation and airway infections are observed. ${ }^{1}$ Major pathogens responsible for infections in CF patients are Staphylococcus aureus and Haemophilus influenzae in early stages of disease and Pseudomonas aeruginosa (first, nonmucoid and then mucoid strains) in later periods. Other opportunistic bacteria such as Stenotrophomonas maltophilia, Burkholderia cepacia complex, Burkholderia gladioli, Achromobacter xylosoxidans, Ralstonia spp., Cupriavidus spp., and Pandoraea spp. can also be responsible for poor outcomes. ${ }^{2}$ P. aeruginosa colonization occurring in most $\mathrm{CF}$ patients is connected with the ability of this microorganism to establish a drug-resistant biofilm. Its formation is facilitated by a reduced activity of bactericidal substances in the CF airway and by the presence of a thick, viscous, and dehydrated mucus. ${ }^{3}$ Although extracellular DNA released from $P$. aeruginosa is required for the initial step of $P$. aeruginosa biofilm formation, further stages are promoted by the pool of DNA derived from host cells, mostly neutrophils. Complementing planktonic $P$. aeruginosa with neutrophil F-actin and/or DNA results in P. aeruginosa attachment to the DNA/F-actin network and biofilm formation. Since almost all eukaryotic cells contain significant amounts of actin, it is possible that in some clinical situations, for example, in severe skin burns, actin and DNA deriving from necrotic epithelial cells will also enhance $P$. aeruginosa biofilm httpsllat (c) (i) (5) $\odot 2018$ Bucki et al. This work is published and licensed by Dove Medical Press limited. The full terms of this license are available at https://www.dovepress.com/terms. BY NG php and incorporate the Creative Commons Attribution - Non Commercial (unported, v3.0) License (http://creativecommons.org/licenses/by-nd/3.0/). By accessing the work you hereby accept the Terms. Non-commercial uses of the work are permitted without any further permission from Dove Medical Press Limited, provided the work is properly attributed. For permission for commercial use of this work, please see paragraphs 4.2 and 5 of our Terms (https://www.dovepress.com/terms.php). 
formation. ${ }^{4}$ During persistent Pseudomonas lung infection that develops in patients suffering from CF by mucoid strains, large quantities of alginate exopolysaccharide used in biofilm formation are expressed, in addition to virulence factors (toxins, hemolysins, and proteases). Alginate accounts for increased Pseudomonas resistance to opsonization, phagocytosis, and digestion and increased resistance to various antibiotics. The eradication of mucoid strains is challenging and often impossible, even with intensive antibiotic treatment. ${ }^{5}$

CF airway secretions differ from those from healthy people and, similar to other purulent fluids, contain high concentrations of DNA and F-actin. Polymeric substances released from necrosing inflammatory cells aggregate, forming large bundles. ${ }^{6,7}$ DNA/F-actin bundles with other anionic macromolecules, such as glycoproteins and extracellular polymers produced by colonizing bacteria, are stabilized by multivalent cations such as histones, antimicrobial peptides, and other positively charged molecules. ${ }^{8}$ Natural antibacterial factors - cathelicidin LL-37, defensins, lactoferrin, and lysozyme - are locally inhibited in purulent fluids because they are trapped in forming bundles. ${ }^{9}$ In addition, the polymeric components present in CF sputum and other purulent body fluids create a network that contributes to its abnormal biophysical properties that prevent its clearance. ${ }^{10}$ Therefore, in treatment of infections in $\mathrm{CF}$ patients, apart from antibiotics (mainly ciprofloxacin, $\beta$-lactams, aminoglycosides), surface liquid hydrators/mucolytics such as nebulized hypertonic saline, inhaled mannitol, and DNase 1, as well as intensive chest physiotherapy, are used. ${ }^{11}$ Although the efficacy of DNase 1 is well established, the utility of some other substances with the potential to reduce the viscoelasticity of airway secretions such as gelsolin or anionic polyamino acids (poly-aspartate or poly-glutamate) has been proposed..$^{8,12}$ The resulting disruption of actin-DNA filaments strongly inhibits biofilm formation and disperses planktonic bacterial cells. Here we evaluate the effect of polyelectrolytes in purulent body fluids on the efficacy of some positively charged conventional antibiotics: tobramycin, colistin, and polymyxin, and an antimicrobial peptide (cathelicidin LL-37) and its lipid mimic (ceragenin CSA-13). Activity was tested in experimental systems containing F-actin and DNA from purulent fluid with and without substances to depolymerize the DNA/F-actin network such as plasma gelsolin, DNase 1 , and poly-aspartate.

\section{Material and methods Materials}

$P$. aeruginosa strain Xen 5 and $S$. aureus Xen30, engineered through conjugation and transposition of a plasmid carrying transposon Tn5 lux CDABE, were purchased from Caliper Life Science Inc. (Waltham, MA, USA). LL-37 (LLGDFFRKSKEKIGKEFKRIVQRIKDFLRNLVPRTES) was purchased from Peptide 2.0 Inc. (Chantilly, VA, USA). Ceragenin CSA-13 was prepared as previously described and was characterized by nuclear magnetic resonance and liquid chromatographymass spectroscopy. ${ }^{13}$ Pseudomonas isolation agar (PIA), Luria-Bertani broth (LB) and tryptic soy broth (TSB) were purchased from DIFCO (Sparks, MD, USA). Pulmozyme (recombinant human deoxyribonuclease 1 - rhDNase 1) was from Genentech Inc. (South San Francisco, CA, USA). Poly(D,L)-aspartic acid (p-ASP), colistin, tobramycin, and deoxyribonucleic acid (DNA) from fish sperm were purchased from Sigma-Aldrich Co. (St Louis, MO, USA). Aztreonam was from AdooQ BioScience (Irvine, CA, USA). CF sputum samples (Table 1) were collected from patients attending the University of Pennsylvania Health System Adult Cystic Fibrosis Center at Presbyterian Hospital (PA, USA; IRB-803255). Within 2 hours of collection, the samples were divided into aliquots containing 500-1000 mg; then the aliquots were flash frozen in liquid nitrogen. To evaluate the antimicrobial agents in the presence of pus (Table 2), the purulent materials were collected in Holy Cross Oncology Center of Kielce, Poland under institutional review board (IRB) approval: R-I-002/575/2013. All pus samples were obtained from adult patients. The study

Table I Demographic characterization of patients providing sputum specimens $(n=8)$

\begin{tabular}{lllll}
\hline Subject & Sex & Age (years) & Type of CF mutation & DNA (mg/g) \\
\hline $001 / 50$ & Male & 55 & DeltaF508/RII7H-7T & 0.66 \\
$002 / 60$ & Female & 27 & DeltaF508X2 & 1.23 \\
$003 / 62$ & Male & 27 & DeltaF508X2 & 7.95 \\
$004 / 72$ & Male & 18 & DeltaF508/unknown & 1.82 \\
$005 / 77$ & Female & 24 & DeltaF508/unknown & 9.74 \\
$006 / 81$ & Male & 28 & DeltaF508X2 & 0.65 \\
$007 / 108$ & Male & 32 & DeltaF508/G542X & 5.16 \\
$008 / 113$ & Female & 51 & DeltaF508/unknown & 2.88 \\
\hline
\end{tabular}

Abbreviation: CF, cystic fibrosis. 
Table 2 Source and DNA concentration of pus samples $(n=6)$

\begin{tabular}{lll}
\hline Specimens & Source of pus & DNA $(\mathbf{m g} / \mathbf{g})$ \\
\hline A & Abdominal fluid & 0.21 \\
B & Synovial fluid & 0.57 \\
C & Pleura fluid & 1.03 \\
D & Carbunculus & 1.52 \\
E & Bile & 0.87 \\
F & Skin abscess & 0.96 \\
\hline
\end{tabular}

was approved by the Institutional Review Board of The Jan Kochanowski University in Kielce. For both the studies, all subjects provided written informed consent.

\section{Bacterial growth}

$P$. aeruginosa Xen5 (PA Xen5) and $S$. aureus Xen30 (SA

Xen30) (methicillin-resistant Staphylococcus aureus [MRSA] strain) were stored in TSB with glycerol (25\%) at $-80^{\circ} \mathrm{C}$. When required, bacteria from frozen stocks were streaked on cetrimide PIA or LB agar plates. In all experiments, bacteria were grown in LB broth.

\section{Antimicrobial testing}

Bacteria were grown overnight to reach a logarithmic phase of growth. The bacterial concentration was measured by spectrophotometry $\left(\mathrm{OD}_{600 \mathrm{~nm}}\right)$. To assess antibacterial activity of LL-37, CSA-13, colistin, tobramycin, and aztreonam in the presence of purulent fluids, the bacteria were added to a 1:10 dilution of $\mathrm{CF}$ sputum or pus made in $0.9 \% \mathrm{NaCl}$. Before the addition of bacteria, the solution was homogenized by vortexing the sample in a $15-\mathrm{mL}$ conical tube with glass beads. Evaluation of chemiluminescence and colony forming units (CFU) was performed from samples treated with $1 \mu \mathrm{g} / \mathrm{mL}$ of p-ASP or its combination with DNase $(1 \mu \mathrm{g} / \mathrm{mL}) .{ }^{14}$

\section{Bacterial biofilm formation}

The biomass of biofilm formed from PA Xen 5 suspended in LB in the presence of cathelicidin LL-37, ceragenin CSA13 , polymyxin $\mathrm{B}$, tobramycin, colistin and aztreonam or their combination with DNase 1, p-ASP, or DNase 1/p-ASP was assessed using crystal violet $(\mathrm{CV})$ staining $(0.1 \%) .{ }^{15}$ In each experiment, an overnight culture of PA Xen5 in LB was diluted to $\sim 10^{5} \mathrm{CFU} / \mathrm{mL}$, bacterial suspensions were placed in 96-well polystyrene plates, and a biofilm was allowed to form for 24 hours. Bacteria adherent to the plate were considered a biofilm, and cells not adherent to the surface of the plate were considered planktonic and were washed out before CV staining measurements were performed.

\section{DNA content in purulent human body} fluids and microscopy of DNA and F-actin bundle structure

DNA concentration was evaluated in manually homogenized samples $(1: 100$ dilution in $0.9 \% \mathrm{NaCl})$ by comparing sample fluorescence after addition of YOYO-1 with a series of known DNA concentration standards using an SL-50B spectrofluorometer (PerkinElmer Inc., Waltham, MA, USA). The morphology of DNA and F-actin structures in pus samples was determined by staining with YOYO-1 and rhodamine phalloidin as described previously. ${ }^{16}$

\section{Evaluation of viscoelasticity}

Bohlin CVO Rheometer (Malvern Instruments, Malvern, UK) was used to assess the viscoelastic properties of eight CF sputum samples, which were determined from the relation between applied force and the resulting deformation in a creep-recovery as described previously. ${ }^{16}$ Prior to treatment with PBS ( $50 \mu \mathrm{L} / \mathrm{g}$ of sputum), PBS containing DNase $(1 \mu \mathrm{g} / \mathrm{g}$ sputum), p-ASP ( $1 \mu \mathrm{g} / \mathrm{g}$ of sputum), or their combination, previously frozen samples were thawed at room temperature for about 30 minutes. Once treated, the samples were placed between the rheometer plates with a gap of $0.8 \mathrm{~mm}$. Water-saturated filter paper surrounded the sample to prevent drying. Creep tests were performed by applying a constant stress of $1 \mathrm{~Pa}$ for 20 seconds, and recovery was measured for 30 seconds after the stress was removed.

\section{Statistical analysis}

Data are reported as mean $\pm \mathrm{SD}$. Differences between means were evaluated using the Student's $t$-test, with $p<0.05$ being taken as the level of significance.

\section{Results \\ DNase I and p-Asp enhance bactericidal activity of antibacterial agents in CF sputum}

Using bioluminescent $P$. aeruginosa Xen5 strain, we determined the influence of the mucolytic compounds DNase 1 and p-ASP, used separately and in combination, on the activity of LL-37, CSA-13, and selected conventional antibiotics (polymyxin B, tobramycin, colistin, and aztreonam) (Figure 1). In this set of experiments, the decrease of luminescence reflects the decreased viability of bacteria. All tested antibacterials showed activity in the presence of CF sputum, but to different extents, as expressed by luminescence values that correspond 
to the amounts of bacteria/metabolic activity. Addition of DNase 1 or $\mathrm{p}$-ASP at $1 \mu \mathrm{g} / \mathrm{mL}$ results in a luminescence decrease, which indicates an increase in the antibacterial activities of the tested agents. The combination of DNase 1 and p-ASP displays a stronger antibacterial effect compared to the effect of individual molecules (Figure 1). A larger
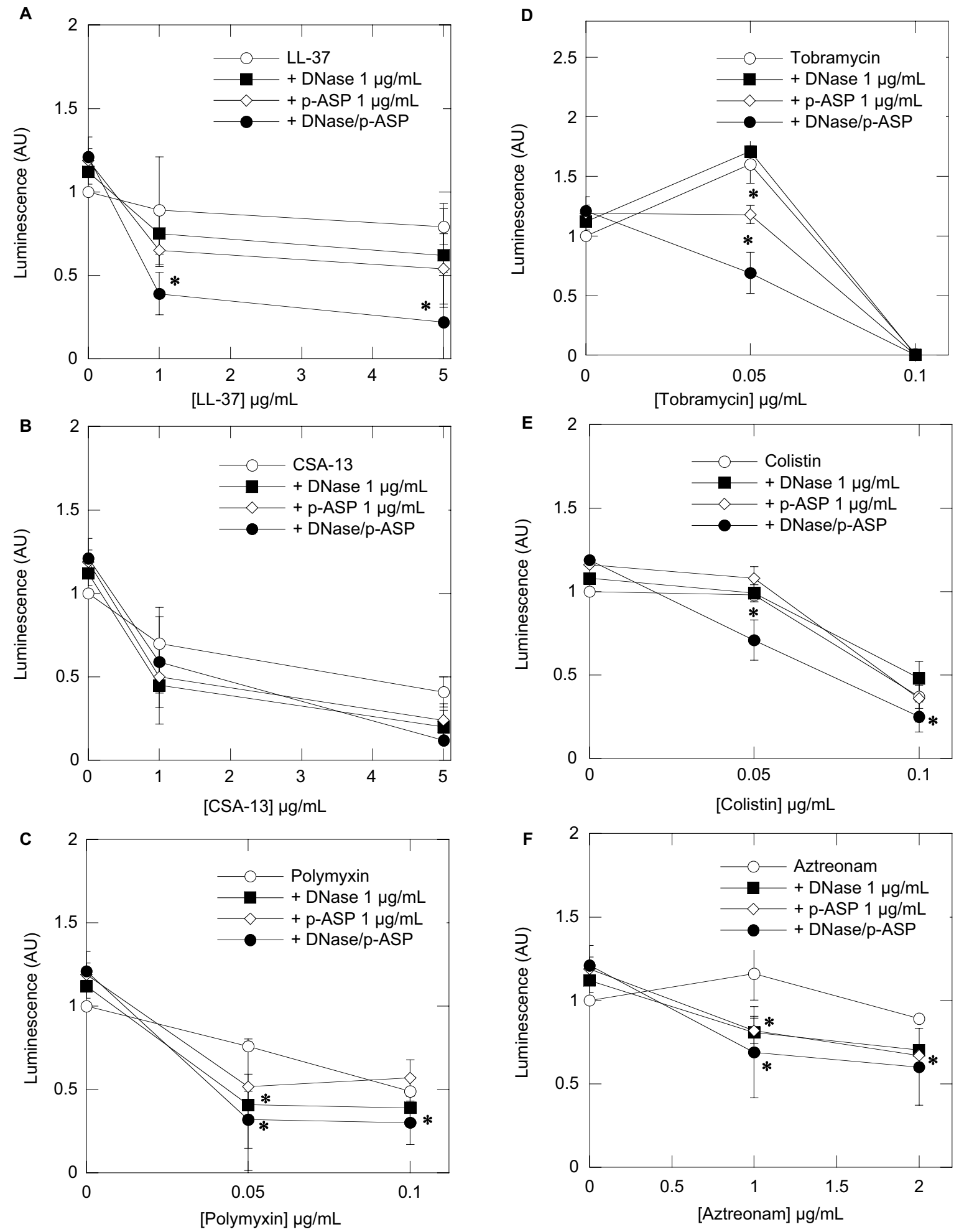

Figure I Decrease of Pseudomonas aeruginosa Xen5 luminescence (indicative of decreased viability) after 8 hours of incubation with cathelicidin LL-37 (A), ceragenin CSA-I 3 $(\mathbf{B})$, polymyxin B $(\mathbf{C})$, tobramycin $(\mathbf{D})$, colistin $(\mathbf{E})$, and aztreonam $(\mathbf{F})$ or their combination with DNase I, P-ASP or DNase I/P-ASP assessed in individual samples of I:I0 dilution of cystic fibrosis sputum. Error bars represent standard deviations from eight different sputum samples ( $n=8)$. *Statistically significant ( $p<0.05$ ) compared to control. Abbreviation: p-ASP, poly-(d,I)-aspartic acid. 
additive effect was observed when a combination of DNase 1 and p-ASP was used with LL-37 peptide compared with CSA-13 (Figure 1). These results are in line with the different mechanisms of action of DNase and p-ASP.

\section{P. aeruginosa Xen 5 biofilm formation is inhibited by the combination of antibacterial agents with DNase I, p-ASP, or DNase I/p-ASP}

To assess the influence of DNase 1, p-ASP, or DNase 1/pASP combination on LL-37, tobramycin, CSA-13, colistin, polymyxin $\mathrm{B}$, and aztreonam activity against $P$. aeruginosa biofilm formation, a stationary biofilm mass was measured after 24 hours of growth. In agreement with the observed effects of DNase 1/p-ASP combination showing improved inhibition of bacterial growth by antibacterial agents, inhibition of biofilm formation was also higher when antimicrobials were added with a mix of DNase 1/p-ASP (Figure 2).

\section{Outgrowth of S. aureus Xen30 from contaminated pus}

Using bacterial outgrowth from pus samples (Table 2), we evaluated the influence of DNase 1, p-ASP, and DNase 1/pASP with the addition of $S$. aureus Xen30 followed by the treatment with ceragenin CSA-13 (Figure 3A and 3C) or LL-37 peptide (Figure 3A and 3B) with or without the addition of DNase 1, p-ASP, or DNase 1/p-ASP in PBS (Figure $3 \mathrm{~B}$ and $3 \mathrm{D}$ ) compared to the activity of antibacterial agents in PBS containing 50\% pus. Comparing these two settings reveals an inhibitory effect of DNase 1 and p-ASP on bacterial outgrowth, especially when used in combination, in samples containing pus, particularly when also combined with LL-37 peptide (Figure 3).

\section{Change of CF sputum viscosity upon treatment with DNase, $\mathrm{p}$-ASP, or their combination}

Using a creep-recovery test, we determined compliance values at 3 seconds under $1 \mathrm{~Pa}$ stress in eight sputum samples collected from adult CF patients (Table 1 and Figure 4). After the addition of DNase $1, \mathrm{p}$-ASP ( $1 \mu \mathrm{g} / \mathrm{g}$ of sputum), or their combination, we observed comparable changes in CF sputum compliance and unrecovered creep showing that DNA/F-actin bundles present in CF sputum are likely partially fluidized. Interestingly, changes in viscoelastic properties achieved with DNase 1 might also be obtained with short, anionic polymers that interfere with the sputum polyelectrolyte network. Combination of DNase 1 with p-ASP had an additive effect (Figure 5).

\section{Discussion}

In advanced stages of $\mathrm{CF}$ and some other pathological conditions, cellular secretions accumulate and reassemble with host cell and pathogen-derived debris into pus. ${ }^{17}$ The large quantity of polymeric substances, mainly DNA and $\mathrm{F}$-actin, form a rigid network that determines the biophysical properties of sputum (viscoelasticity and adhesiveness) and strongly influences the course of the disease and the mode of treatment. ${ }^{18}$ As most of the clinically used antibiotics are positively charged to target the bacterial outer wall, their interaction with the polyanionic network can diminish their activity and cause entrapment within the network. ${ }^{19}$ Indeed, the potency of natural antimicrobial cationic peptides present in airway fluids is inhibited by DNA/F-actin bundles. ${ }^{14,20}$ Standard strategies used in CF treatment, the fluidization of the sputum by a recombinant human deoxyribonuclease (rhDNase) and using antibiotics in high doses in a systemic and aerosolized form can at least partially overcome the inhibiting influence of a polyanionic network. ${ }^{21}$ Although the utility of rhDNase significantly improves lung function, not all patients with CF benefit from this treatment. ${ }^{22}$ Therefore, there is a continuous need for new effective therapies.

In this study, we assessed the influence of clinically used DNase 1 and a new mucolytic substance, p-ASP, on antiPseudomonas activity of conventional antibiotics (polymyxin, tobramycin, colistin, and aztreonam) measured in a pus environment. Both the mucolytics, when used individually, raised the activity of the tested antibacterial agents, but their combined effect was much stronger, indicating the existence of an additive effect that might be based on the different modes of action of these agents. The ability of DNase 1 to depolymerize DNA is relatively low in condensed DNA aggregates, and p-ASP's ability to disperse DNA-containing bundles increases the activity of DNase $1 .{ }^{16}$ These results are consistent with our previous data, and other reports showing similar anti-Pseudomonas activity of cathelicidin LL-37 first inhibited by polyanions and then restored by the addition of a substance with mucolytic properties, such as the F-actin severing protein gelsolin. ${ }^{16,19}$ The larger effect displayed by LL-37 compared to CSA-13 underlines the differences in the physicochemical structures of these compounds (total positive charge, density, and distribution). CSA-13 is a steroid molecule with smaller size and lower positive charge and is much more resistant to inactivation by F-actin or DNA compared 

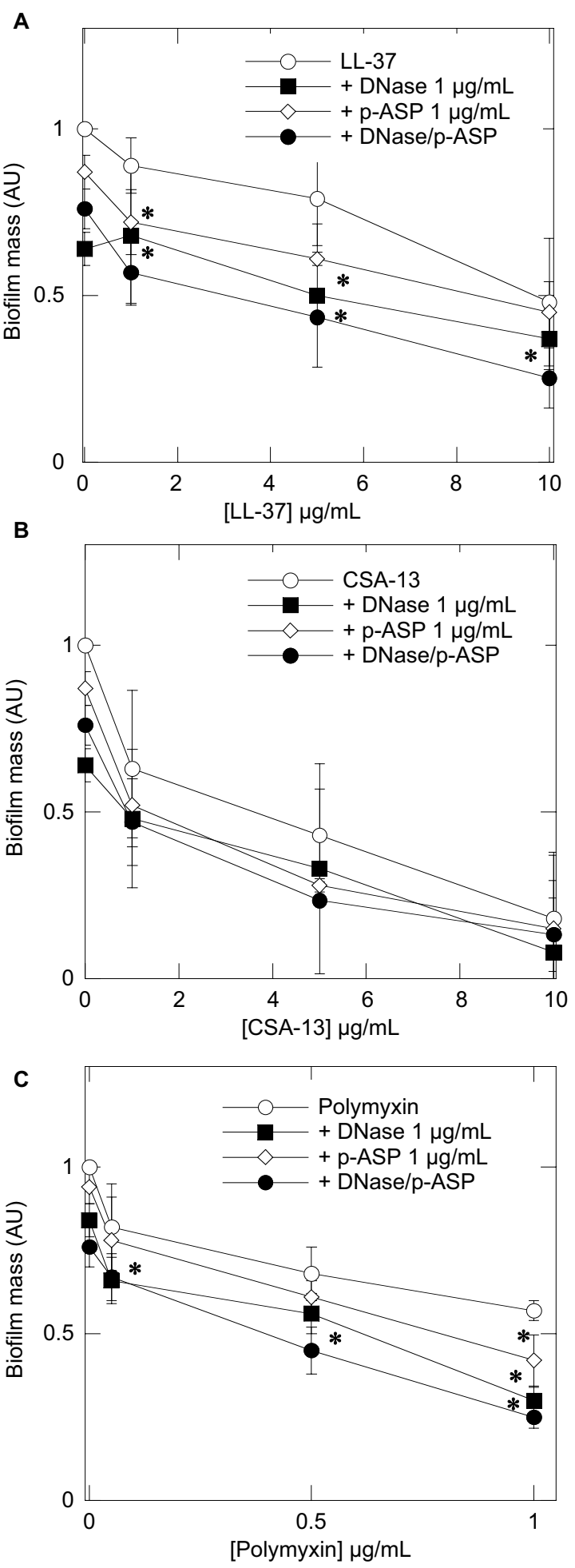

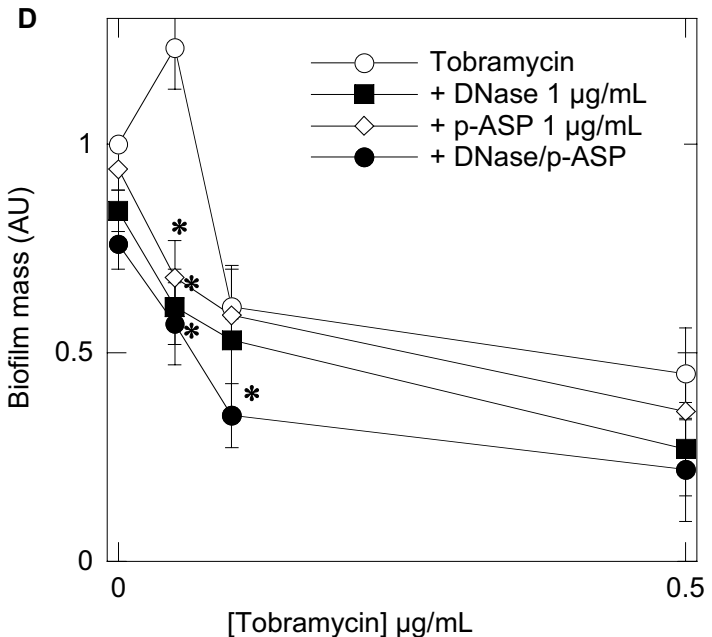

E
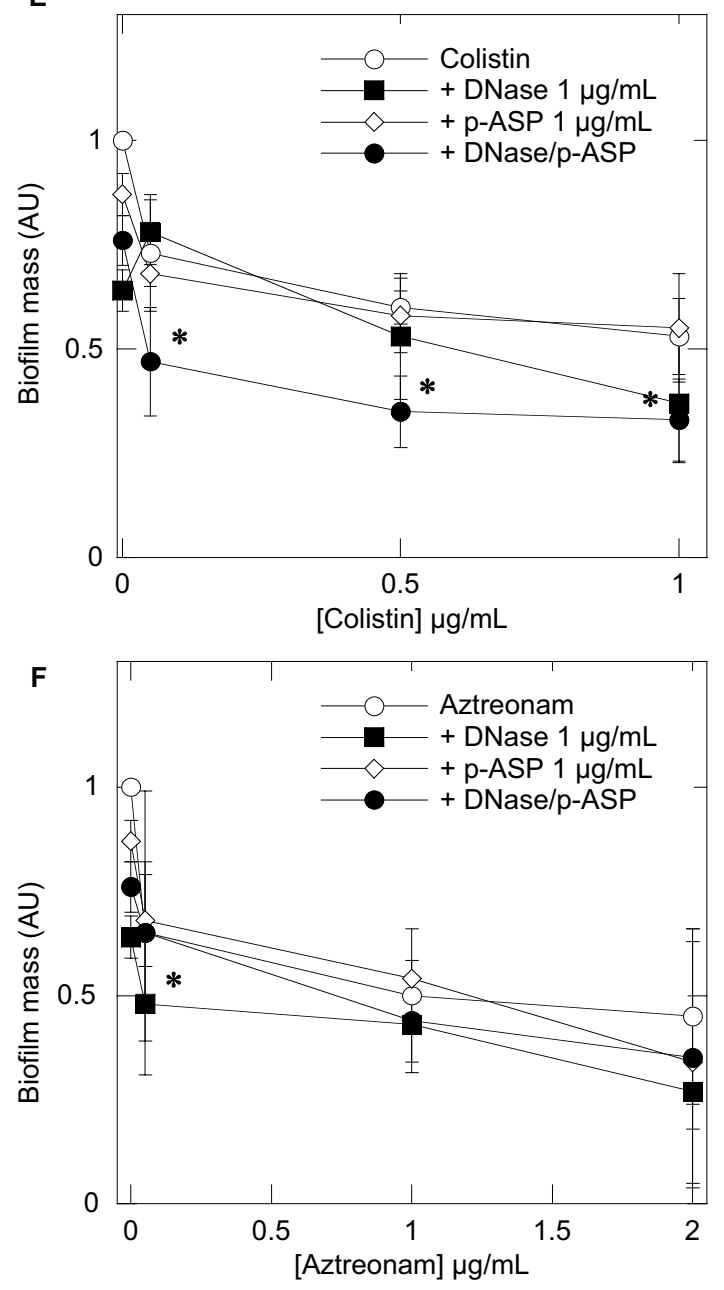

Figure 2 Pseudomonas aeruginosa Xen5 biofilm mass formed after 24 hours growth in LB medium containing cathelicidin LL-37 (A), ceragenin CSA- I3 (B), polymyxin B (C), tobramycin (D), colistin (E), and aztreonam (F) or their combination with DNase I, p-ASP, or DNase I/p-ASP. Error bars represent standard deviations from three to five measurements. *Statistically significant $(p<0.05)$ compared to control.

Abbreviations: $\mathrm{p}-\mathrm{ASP}$, poly-(d,I)-aspartic acid; LB, Luria-Bertani broth.

to LL-37 peptide. Possessing both antimicrobial and antiinflammatory properties, ceragenins could be considered as a candidate for developing new CF treatments. ${ }^{23}$ The successful treatment of lung infections in CF must address not only the changed physicochemical properties of airway secretions but also the capacity of pathogens to form a biofilm. 

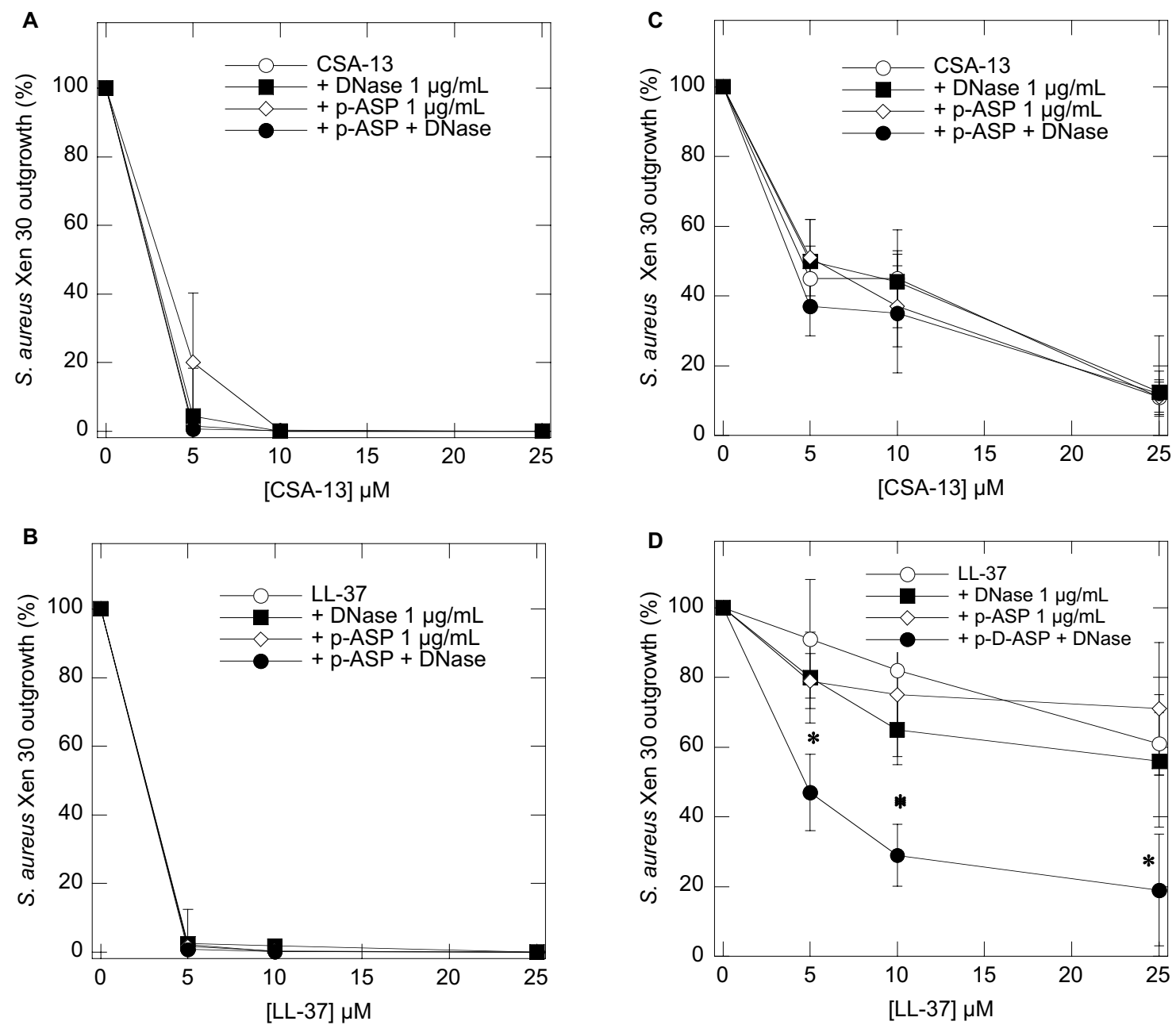

Figure 3 Bacterial outgrowth from PBS (A and B) or PBS containing 50\% pus (C and D) that were infected with S. aureus Xen30 and treated with ceragenin CSA-13 (A and $\mathbf{C}$ ) or LL-37 peptide (B and $\mathbf{D})$ with or without the presence of DNase I, p-ASP, or DNase I/p-ASP. Error bars represent standard deviations from six different pus samples $(\mathrm{n}=6)$. *Statistically significant $(p<0.05)$ compared to control.

Abbreviations: p-ASP, poly-(d,I)-aspartic acid; S. aureus, Staphylococcus aureus.

Tobramycin, the most widely used antibiotic in the treatment of pulmonary infection in CF patients, is able to prevent biofilm formation and disrupt existing biofilms. Aztreonam, another antimicrobial agent clinically available in parenteral and inhaled forms, seems to be less effective, and its anti-biofilm activity is variable depending on bacterial strain. ${ }^{24}$ Because biofilm establishment is facilitated by host components such as neutrophil-derived polyanions, depolymerizing substances may enhance the anti-biofilm activity of antibiotics, especially those with a positive charge. DNase 1 and p-ASP used in combination disrupt $P$. aeruginosa biofilm formation more effectively than when used individually. ${ }^{25}$ In agreement with previous experiments, we have observed that DNase 1 and p-ASP when administrated together increase antibiofilm activity of the antibiotics polymyxin, tobramycin, colistin, and aztreonam. ${ }^{26}$ This hypothesis is strongly supported by observed changes in viscoelastic properties of CF sputum upon treatment with sputum-fluidizing agents (Figure 5). Other studies show an inhibitory role of DNase 1 and p-ASP in the adhesion of oral bacteria and in the development of dental plaque, as well as the ability to inhibit $P$. aeruginosa biofilm formation on contact lenses..$^{27,28}$

Since airway secretions in $\mathrm{CF}$ are similar to pus formed as a consequence of other microbial infections, the DNA/Factin polyanionic network should be considered as a potential new therapeutic target in various clinical situations associated with biofilm formation or/and the accumulation of pus. The second part of our experiments demonstrated that mucolytic substances added to $S$. aureus outgrowing from PBS containing $50 \%$ pus enhance the activity of ceragenin CSA-13 and LL-37 peptide. These data show that fluidization of pus by DNase 1 or p-ASP could be used practically to improve the treatment 

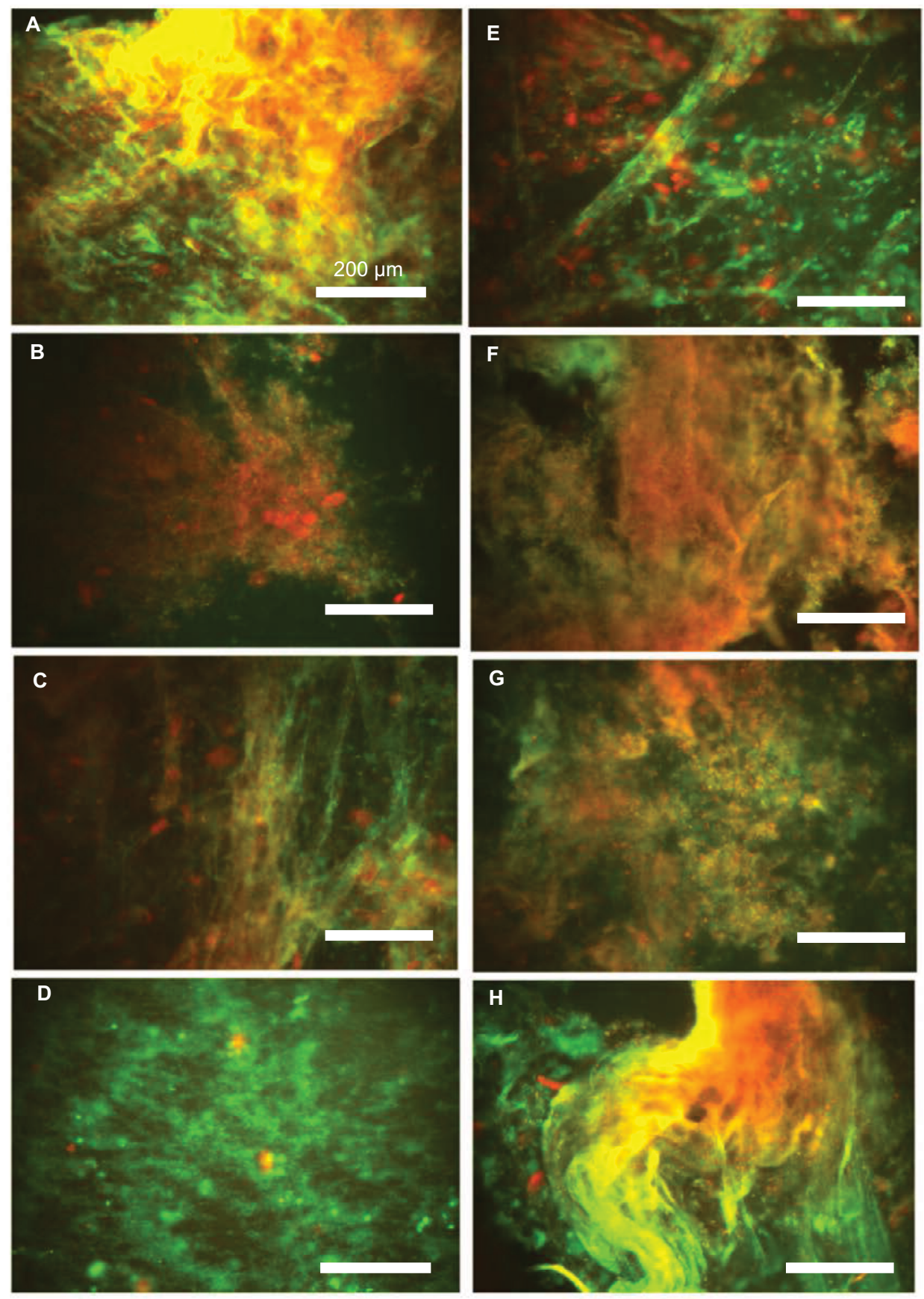

Figure 4 Overlap of fluorescence images of DNA (green)/F-actin (red) staining $(\mathbf{A}-\mathbf{H})$ in eight individual pus samples (Table I). Data from one representative experiment are shown.

of some infections, for example, purulent skin and soft-tissue infections. We have chosen S. aureus as the target pathogen because it is the main etiological agent of such infections. Good antibiotic penetration into the site of infection and sufficient drug concentration is crucial to achieve a favorable clinical outcome. Apart from mucolytic substances, other treatment strategies have been investigated. One of them is the possibility of using antibiotics entrapped in liposomes, which can protect antibacterial agents from inhibitory effects of DNA/F-actin complexes. ${ }^{29}$ The concept that a polyelectrolyte network in purulent body fluids can be a potential new therapeutic target seems to be worth further investigation, especially in the era of emerging antibiotic resistance and lack of new antibacterial drugs. Although the role of DNase is well established and some new mucolytic substances are examined, some topics need future exploration. The influence of DNA depolymerizing agents on the immune response to bacterial and fungal pathogens, which involves neutrophil extracellular trap formation and TLR 9 activation, might also be affected by such agents. In addition, this relatively new pathogen-killing mechanism might 


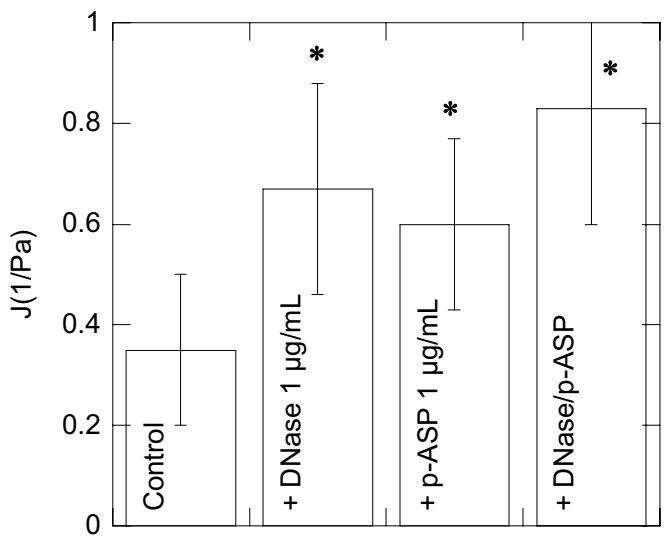

Samples

Figure 5 Viscolelastic properties of CF sputum samples upon I Pa shear stress, after I hour of incubation with DNase I, p-ASP, or their combination. Error bars represent standard deviations from eight different sputum samples $(n=8)$. * Statistically significant $(p<0.05)$ compared to untreated specimens. Abbreviations: CF, cystic fibrosis; p-ASP, poly-(d,I)-aspartic acid.

require a temporal structure composed of the extracellular nuclear DNA, granular antimicrobial peptides, and nuclear and cytoplasmic proteins released from some immunocompetent cells. This structure can most likely be affected by depolymerizing substances; hence, the immune response may be inhibited in some settings. ${ }^{30}$

\section{Acknowledgments}

This work was supported by the National Science Center, Poland under Grant UMO-2015/17/B/NZ6/03473 (to RB) and by NIH P01 GM096971 (to PAJ).

\section{Disclosure}

The authors report no conflicts of interest in this work.

\section{References}

1. Heijerman H. Infection and inflammation in cystic fibrosis: a short review. J Cyst Fibros. 2005;4(Suppl 2):3-5.

2. Lipuma JJ. The changing microbial epidemiology in cystic fibrosis. Clin Microbiol Rev. 2010;23(2):299-323.

3. Moreau-Marquis S, Stanton BA, O’Toole GA. Pseudomonas aeruginosa biofilm formation in the cystic fibrosis airway. Pulm Pharmacol Ther. 2008;21(4):595-599.

4. Walker TS, Tomlin KL, Worthen GS, et al. Enhanced Pseudomonas aeruginosa biofilm development mediated by human neutrophils. Infect Immun. 2005;73(6):3693-3701.

5. Pritt B, O'Brien L, Winn W. Mucoid Pseudomonas in cystic fibrosis. Am J Clin Pathol. 2007;128(1):32-34.

6. Sheils CA, Käs J, Travassos W, et al. Actin filaments mediate DNA fiber formation in chronic inflammatory airway disease. Am J Pathol. 1996;148(3):919-927.

7. Rubin BK. Mucus structure and properties in cystic fibrosis. Paediatr Respir Rev. 2007;8(1):4-7

8. Tang JX, Wen Q, Bennett A, et al. Anionic poly(amino acid)s dissolve F-actin and DNA bundles, enhance DNase activity, and reduce the viscosity of cystic fibrosis sputum. Am J Physiol Lung Cell Mol Physiol. 2005;289(4):L599-L605.
9. Bals R, Weiner DJ, Meegalla RL, Accurso F, Wilson JM. Saltindependent abnormality of antimicrobial activity in cystic fibrosis airway surface fluid. Am J Respir Cell Mol Biol. 2001;25(1):21-25.

10. Broughton-Head VJ, Smith JR, Shur J, Shute JK. Actin limits enhancement of nanoparticle diffusion through cystic fibrosis sputum by mucolytics. Pulm Pharmacol Ther. 2007;20(6):708-717.

11. Bhatt JM. Treatment of pulmonary exacerbations in cystic fibrosis. Eur Respir Rev. 2013;22(129):205-216.

12. Davoodian K, Ritchings BW, Ramphal R, Bubb MR. Gelsolin activates DNase I in vitro and cystic fibrosis sputum. Biochemistry. 1997;36(32):9637-9641.

13. Ding B, Guan Q, Walsh JP, et al. Correlation of the antibacterial activities of cationic peptide antibiotics and cationic steroid antibiotics. $J$ Med Chem. 2002;45(3):663-669.

14. Bucki R, Namiot DB, Namiot Z, Savage PB, Janmey PA. Salivary mucins inhibit antibacterial activity of the cathelicidin-derived LL-37 peptide but not the cationic steroid CSA-13. JAntimicrob Chemother. 2008;62(2):329-335.

15. Peeters E, Nelis HJ, Coenye T. Comparison of multiple methods for quantification of microbial biofilms grown in microtiter plates. J Microbiol Methods. 2008;72(2):157-165.

16. Bucki R, Cruz K, Pogoda K, et al. Enhancement of Pulmozyme activity in purulent sputum by combination with poly-aspartic acid or gelsolin. J Cyst Fibros. 2015;14(5):587-593.

17. Voynow JA, Rubin BK. Mucins, mucus, and sputum. Chest. 2009; 135(2):505-512.

18. Rubin BK. Secretion properties, clearance, and therapy in airway disease. Transl Respir Med. 2014;2:6.

19. Weiner DJ, Bucki R, Janmey PA. The antimicrobial activity of the cathelicidin LL37 is inhibited by F-actin bundles and restored by gelsolin. Am J Respir Cell Mol Biol. 2003;28(6):738-745.

20. Perks B, Shute JK. DNA and actin bind and inhibit interleukin-8 function in cystic fibrosis sputa: in vitro effects of mucolytics. Am J Respir Crit Care Med. 2000;162(5):1767-1772.

21. Edmondson C, Davies JC. Current and future treatment options for cystic fibrosis lung disease: latest evidence and clinical implications. Ther Adv Chronic Dis. 2016;7(3):170-183.

22. Pressler T. Review of recombinant human deoxyribonuclease (rhDNase) in the management of patients with cystic fibrosis. Biologics. 2008;2(4):611-617.

23. Bucki R, Sostarecz AG, Byfield FJ, Savage PB, Janmey PA. Resistance of the antibacterial agent ceragenin CSA-13 to inactivation by DNA or F-actin and its activity in cystic fibrosis sputum. $J$ Antimicrob Chemother. 2007;60(3):535-545.

24. Yu Q, Griffin EF, Moreau-Marquis S, Schwartzman JD, Stanton BA, O'Toole GA. In vitro evaluation of tobramycin and aztreonam versus Pseudomonas aeruginosa biofilms on cystic fibrosis-derived human airway epithelial cells. J Antimicrob Chemother. 2012;67(11): 2673-2681.

25. Parks QM, Young RL, Poch KR, Malcolm KC, Vasil ML, Nick JA Neutrophil enhancement of Pseudomonas aeruginosa biofilm development: human F-actin and DNA as targets for therapy. J Med Microbiol. 2009;58(Pt 4):492-502.

26. Kaplan JB, LoVetri K, Cardona ST, et al. Recombinant human DNase I decreases biofilm and increases antimicrobial susceptibility in staphylococci. J Antibiot (Tokyo). 2012;65(2):73-77.

27. Guan YH, Lath DL, Graaf T, Lilley TH, Brook AH. Moderation of oral bacterial adhesion on saliva-coated hydroxyapatite by polyaspartate. J Appl Microbiol. 2003;94(3):456-461.

28. Robertson DM, Parks QM, Young RL, et al. Disruption of contact lensassociated Pseudomonas aeruginosa biofilms formed in the presence of neutrophils. Invest Ophthalmol Vis Sci. 2011;52(5):2844-2850.

29. Alipour M, Suntres ZE, Halwani M, Azghani AO, Omri A. Activity and interactions of liposomal antibiotics in presence of polyanions and sputum of patients with cystic fibrosis. PLoS One. 2009;4(5):e5724.

30. Kruger P, Saffarzadeh M, Weber AN, et al. Neutrophils: Between host defence, immune modulation, and tissue injury. PLoS Pathog. 2015;11(3):e1004651. 


\section{Publish your work in this journal}

Infection and Drug Resistance is an international, peer-reviewed openaccess journal that focuses on the optimal treatment of infection (bacterial, fungal and viral) and the development and institution of preventive strategies to minimize the development and spread of resistance. The journal is specifically concerned with the epidemiology of antibiotic resistance and the mechanisms of resistance development and diffusion in both hospitals and the community. The manuscript management system is completely online and includes a very quick and fair peerreview system, which is all easy to use. Visit http://www.dovepress.com/ testimonials.php to read real quotes from published authors.

Submit your manuscript here: https://www.dovepress.com/infection-and-drug-resistance-journal 